\title{
A Serious Game for Exploring and Training in Participatory Management of National Parks for Biodiversity Conservation: Design and Experience
}

\author{
Eurico Vasconcelos ${ }^{1}$ Gustavo Melo ${ }^{2}$ Jean-Pierre Briot ${ }^{3} \quad$ Vinícius Sebba Patto $^{3}$ \\ Alessandro Sordoni $^{3} \quad$ Marta Irving $^{2} \quad$ Isabelle Alvarez $^{3} \quad$ Carlos Lucena $^{1}$ \\ ${ }^{1}$ Pontifícia Universidade Católica (PUC-Rio), Computer Science Department, RJ, Brazil \\ ${ }^{2}$ Universidade Federal do Rio de Janeiro (UFRJ), EICOS Program, RJ, Brazil \\ ${ }^{3}$ Universitè Pierre et Marie Curie, Laboratoire d'Informatique de Paris 6 (LIP6), CNRS, France
}

\begin{abstract}
In this paper, we discuss the experience in the design, use and evaluation of a serious game about participatory management of national parks for biodiversity conservation and social inclusion. Our objective is to help various stakeholders (e.g., environmentalist NGOs, communities, tourism operators, public agencies, and so on) to collectively understand conflict dynamics for natural resources management and to exercise negotiation management strategies for protected areas, one of the key issues linked to biodiversity conservation in national parks. Our serious game prototype combines, techniques such as: distributed role-playing games, support for negotiation between players, and insertion of various types of artificial agents (decision making agents, virtual players, assistant agents). After a general introduction to the project, we will present project's current prototype architecture and results from game sessions, as well as some prospects for the future, namely: the design of assistant artificial agents and of virtual players and the integration of a viability-based simulation engine.
\end{abstract}

Keywords: Serious games, Simulation, Participatory management of Parks

\section{Introduction}

Serious Games [Michael and Chen 2006] are getting increased attention as a novel and effective approach for training and exploring possibilities, in context but without high costs or risks. Indeed, games are a good substitute for direct experience from real world or real infrastructures because they can generate learning experiences in a relatively fast and safe manner [Warmerdan et al. 2006].

In this paper, we will discuss our experience in the design of a serious game about participatory management of national parks for biodiversity conservation and social inclusion. Its main objective is to serve as an epistemic/educational tool. In this game, humans play some role and discuss, negotiate and take decisions about a common domain, in our case environment management decisions. More precisely, the idea is to help park managers, stakeholders and all researchers involved in park management, to explore and train about conflict identification, negotiation and decision strategies for management of parks, with the various perspectives involved, such as: biodiversity conservation, social inclusion and sustained development. This research project, named SimParc (which stands in French for "Simulation Participative de Parcs"), was started in 2006 in order to investigate the use of advanced computer techniques and methodologies (such as serious games) for participatory management of protected areas, more specifically national parks. The current SimParc serious game prototype is based on a role-playing game and computer techniques such as: distributed role-playing interfaces, negotiation support and artificial decision makers. Although intended, primary, to serve as epistemic tool, SimParc also has as ingredients the playfulness and the challenge of a game, presenting funny and interactive maps, negotiations and decision making in different and challenging phases.

\section{The SimParc Project}

\subsection{Project Motivation}

A significant challenge involved in biodiversity management is the management of protected areas (e.g., national parks), which usually undergo various pressures on resources, use and access, which results in many conflicts. This makes the issue of conflict resolution a key issue for the participatory management of protected areas [Irving 2006]. Methodologies intending to facilitate this process are being addressed via bottom-up approaches that emphasize the role of local actors. Examples of social actors involved in these conflicts are: park managers, local communities at the border area, tourism operators, public agencies and NGOs. Examples of inherent conflicts connected with biodiversity protection in the area are: irregular occupation, inadequate tourism exploration, water pollution, environmental degradation and illegal use of natural resources. 
Our SimParc project focuses on participatory parks management. It is based on the observation of several case studies in Brazil. However, we chose not to reproduce exactly a real case, in order to leave the door open for broader game possibilities. Our project aim is to help various stakeholders at collectively understand conflicts and negotiate strategies for handling them.

\subsection{Related Work}

Our initial inspiration was the ComMod approach about participatory methods to support negotiation and decision-making for participatory management of renewable resources [Barreteau 2003]. They pioneer method, called MAS/RPG, consists in coupling multiagent simulation (MAS) of the environment resources and role-playing games (RPG) by the stakeholders [Barreteau 2003]. The RPG acts like a "social laboratory", because players of the game can try many possibilities, without real consequences.

Recent works proposed further integration of roleplaying into simulation, and the insertion of artificial agents, as players or as assistants. Participatory simulation and its standard bearer, the Simulación framework [Guyot and Honiden 2006], focused on a distributed support for role-playing and negotiation between human players. All interactions are recorded for further analysis (thus opening the way to automated acquisition of behavioral models) and assistant agents are provided to assist and suggest strategies to the players. The Games and Multi-Agent-based Simulation (GMABS) methodology focused on the integration of the game cycle with the simulation cycle [Adamatti et al. 2007]. It also innovated in the possible replacement of human players by artificial players. One of our objectives is to try to combine their respective merits and to further explore possibilities of computer support.

\section{The SimParc Role-Playing Game}

\subsection{Game Objectives}

Current SimParc game has an epistemic objective: to help each participant discover and understand in a playful way the various factors, conflicts and the importance of dialogue for a more effective management of parks. Note that this game is not (or at least not yet) aimed at decision support (i.e., we do not expect the resulting decisions to be directly applied to a specific park).

The game is based on a negotiation process that takes place within the park council. This council, of a consultative nature, includes representatives of various stakeholders (e.g., community, tourism operator, environmentalist, nongovernmental association, water public agency). The actual game focuses on a discussion within the council about the "zoning" of the park, i.e. the decision about a desired level of conservation (and therefore, use) for every sub-area (also named "landscape unit") of the park. We consider nine pre-defined potential levels (that we will consider as types) of conservation/use, from more restricted to more flexible use of natural resources, as defined by the (Brazilian) federal bureau of environment management. Examples are: Intangible, the most conservative use, Primitive and Recuperation.

The game considers a certain number of players' roles, each one representing a certain stakeholder. Depending on its profile and the elements of concerns in each of the landscape units (e.g., tourism spot, people, endangered species...), each player, as in a RPG has to embody the designed/selected role with its respective postures and objectives. To facilitate the incorporation of the role by the player, SimParc offers a set of personas to represent him/her during the game (Figure 1). Based on the role, the player will try to influence the decision about the type of conservation for each landscape unit. It is clear that conflicts of interest will quickly emerge, leading to various strategies of negotiation (e.g., coalition formation, trading mutual support for respective objectives, etc).
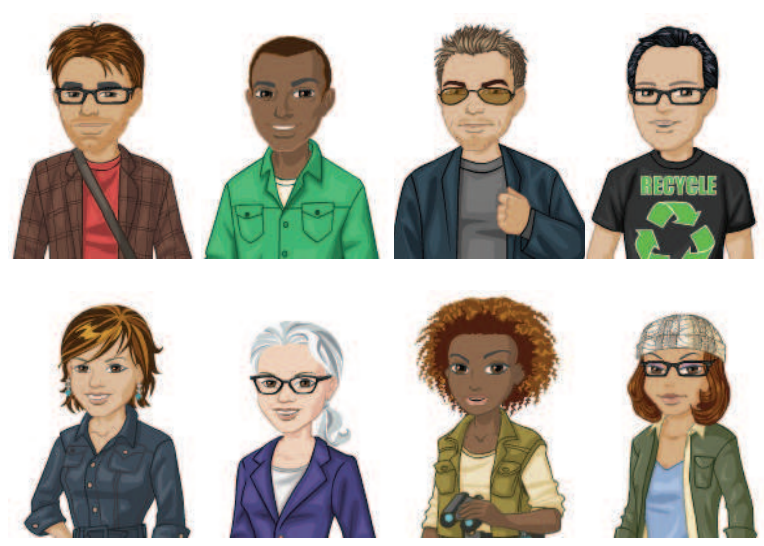

Figure 1: Some examples of personas offered in SimParc.

A special role in the game is the park manager. He is a participant of the game, but as an arbiter and decision maker, and not as a direct player during negotiation and interaction phase. He observes the negotiation taking place between players and takes the final decision about the types of conservation for each landscape unit. His decision is based on the legal framework, on the negotiation process between the players, and on his personal profile (e.g., more conservationist or more open to social concerns) [Irving 2006]. He may also have to explain his decision, closing the game cycle. The park manager may be played by a human or by an artificial agent (see Section 6).

\subsection{Game Cycle}

The game is structured along six steps, as illustrated in Figure 2. At the beginning (step 1), each participant is associated to a role (randomly selected, selected by the administrator or by the participant him/herself.). Then, 


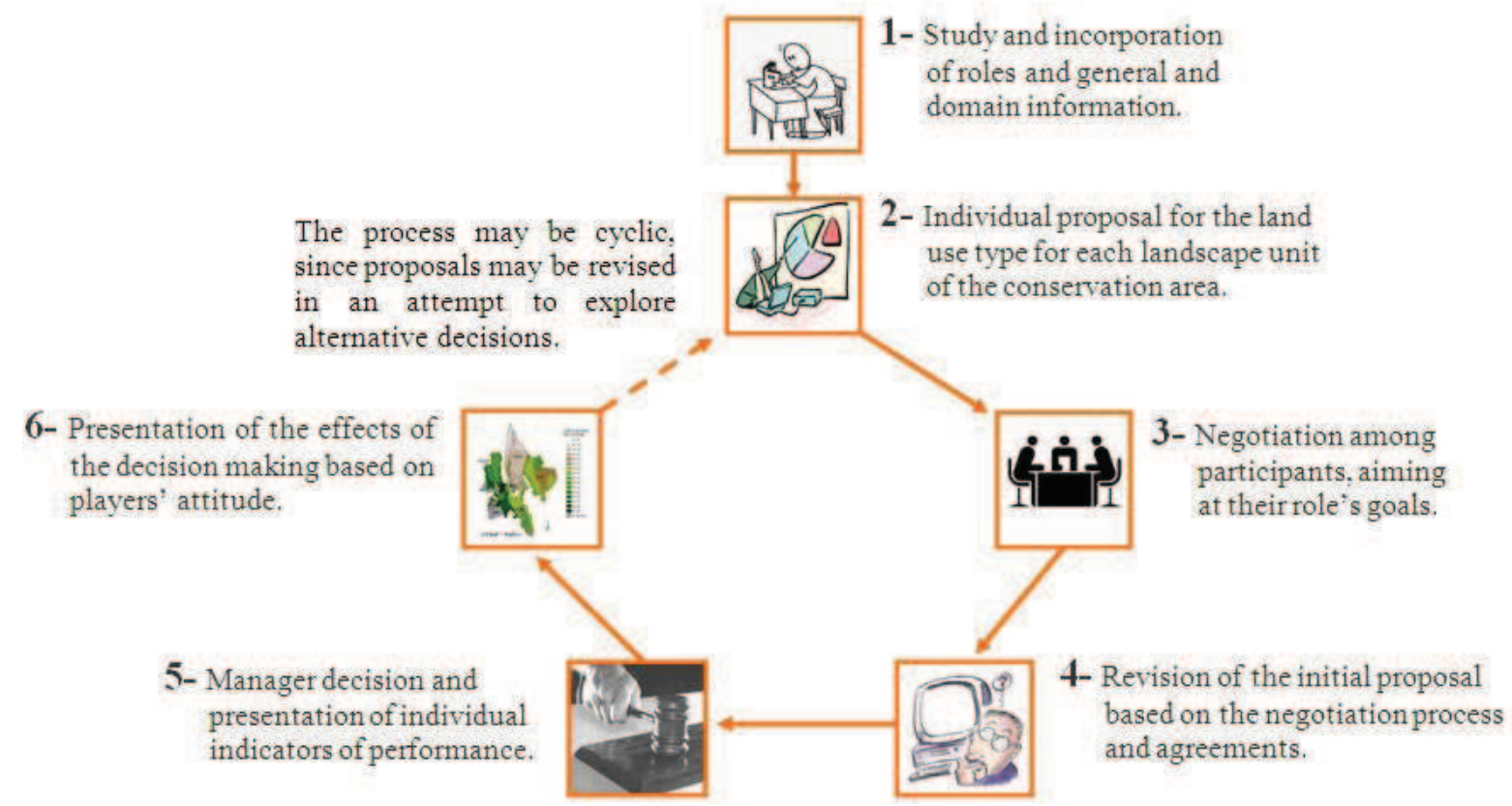

Figure 2: The six steps of the SimParc game.

an initial scenario is presented to each player, including the setting of the landscape units, the possible types of use and the general objective associated to his role. This information is present on a map of a fictitious park that should be "traveled" by the players, by clicking in the elements presented in the map. Then (step 2), each player decides a first proposal of types of use for each landscape unit, based on his/her understanding of the objective of his/her role and on the initial setting. Once all players have done so, each player's proposal is made public.

In step 3, players start to interact and to negotiate on their proposals. This step is, in our opinion, the most important one, where players collectively build their knowledge by means of an argumentation process. In step 4, they revise their proposals and commit themselves to a final proposal for each landscape unit. In step 5, the park manager makes the final decision, considering the negotiation process, the final proposals and also his personal profile (e.g., more conservationist or more sensitive to social issues). Each player can then consult various indicators of his/her performance (e.g., closeness to his initial objective, degree of consensus, etc.). He can also ask for an explanation about the park manager decision rationales.

The last step (step 6) "closes" the epistemic cycle by considering the possible effects of the decision. In the current game, the players provide a simple feedback on the decision by indicating their level of acceptance of the decision. ${ }^{1}$

A new negotiation cycle may then start, thus creating a kind of learning cycle [Kolb 1984]. The

\footnotetext{
${ }^{1}$ A future plan is to introduce some evaluation of the quality of the decision. See Section 7.3.
}

main objectives are indeed for participants: to understand the various factors and perspectives involved and how they are interrelated; to negotiate; to try to reach a group consensus; and to understand cause-effect relations based on the decisions.

\section{The SimParc Game Support Architecture}

Our current prototype benefited from our previous experiences (game sessions and a first prototype) and has been based on a detailed design process. Based on the system requirements, we adopted Web-based technologies (more precisely J2EE and JSF) that support the distributed and interactive character of the game as well as an easy deployment.

Figure 3 shows the general architecture and communication structure of SimParc prototype version 2 . In this second prototype, distributed users (the players and the park manager) interact with the system mediated internally by communication broker agents (CBA). The function of a CBA is to abstract the fact that each role may be played by a human or by an artificial agent. For each human player, there is also an assistant agent offering assistance during the game session.

During the negotiation phase, players (human or artificial) negotiate among themselves to try to reach an agreement about the type of use for each landscape unit (sub-area) of the park. We include below two screen dumps to provide a quick idea about current interface support and their look and feel. The interface for negotiation is shown at Figure 4. It includes advanced support for negotiation (rhetorical markers and dialogue filtering/structuring mechanisms, see details in [Vasconcelos et al. 2009]), access to different 


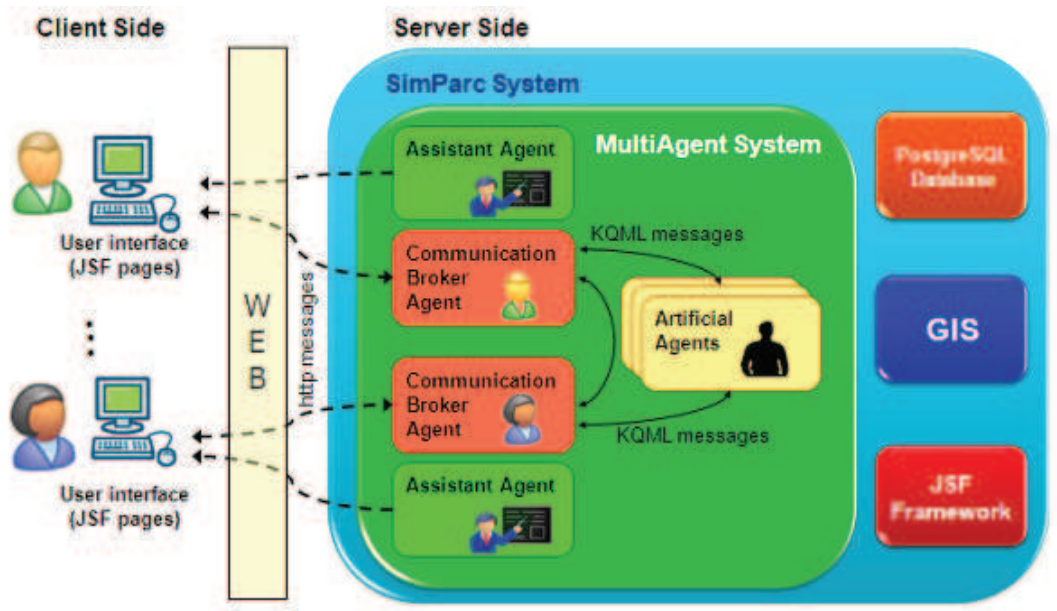

Figure 3: SimParc version 2 general architecture.

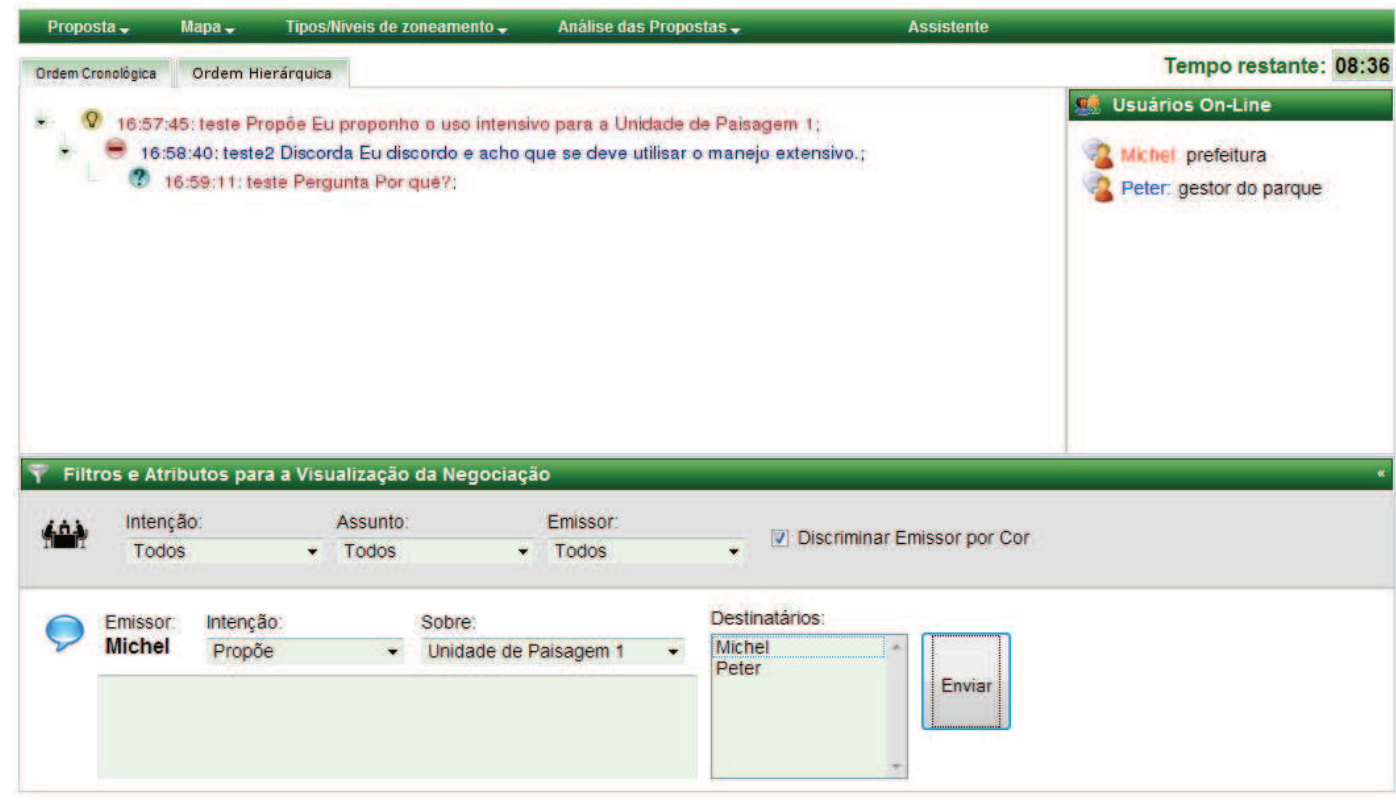

Figure 4: Current prototype's negotiation graphical user interface.
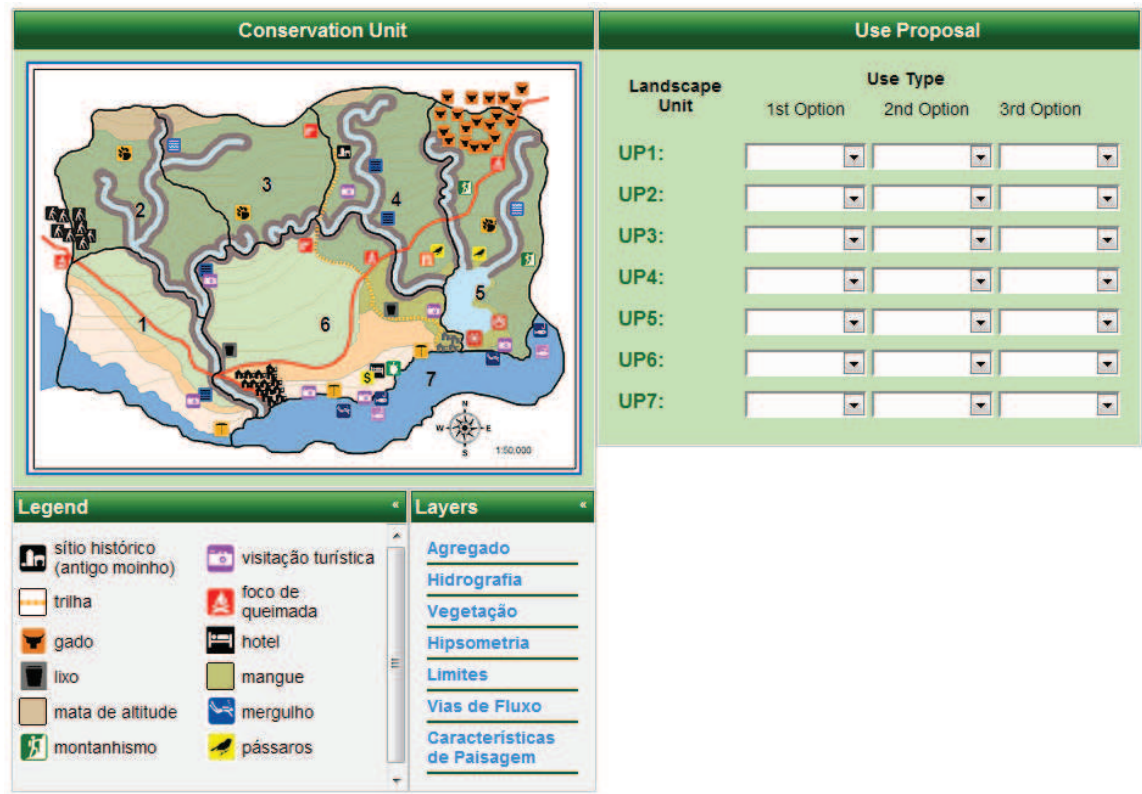

Figure 5: Current prototype's decision graphical user interface. 
kinds of information about other players, land, law and the help of the personal assistant. The negotiation and its interface are detailed in [Vasconcelos et al. 2009]. The interface for players' decision about the types of use is shown at Figure 5. In the interface, the players can analyze the area based in its different layers (e.g. land, hydrography, vegetation...).

A Geographical Information System (GIS) offers to users different layers of information (such as flora, fauna and land characteristics) about the park geographical area. All the information exchanged during negotiation phase, namely users' logs, game configurations, game results and general management information are recorded and read from a PostgreSql database. Report functionality also allows the game manager to get structured information about each game session.

\section{Preliminary Evaluation}

The current computer prototype has been tested through two game sessions by domain expert players (including a professional park manager) in January 2009 (see Figure 6). The 9 roles of the game and the park manager were played by humans. Among these 10 human players, 8 were experts in park management (researchers and professionals, one being a professional park manager in Brazil). The two remaining players were not knowledgeable in park management. One was experienced in games (serious games and video games) and the other one a complete beginner in all aspects.

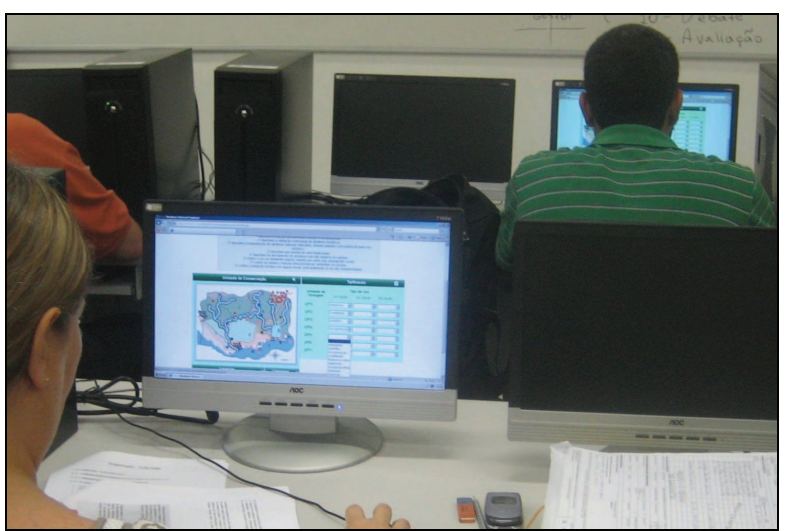

Figure 6: SimParc current prototype game session (2009).

Two aspects of the game were positively evaluated by the participants of the game session: the structure, (script, rules and set tasks) and content (scenes, conflicts, environmental management). Through successful integration of structure and content, SimParc was evaluated as a game that reached the goal of creating, in fact, a "virtual arena" of management. Although the game does not constitute a tool for decision-making directly applicable to real parks, but only a support for epistemic and pedagogical goals, it was highlighted as a positive aspect the proximity of SimParc as a virtual scenario with the reality of park management, making it more attractive for those people working directly in real parks.

In the analysis of the test, two key aspects to the improvement of the game play were highlighted: information and interaction. About information, were considered the conditions for access to content, form, data quality, the quantity of information available, among others. Regarding interaction, were mainly considered the resources and tools available to help players negotiate.

The information is certainly the key to support the SimParc game. Mainly because the game is structured through the use of different terminologies, that in the background are the basis for negotiation between the players. The large volume, complexity of information and conflicts illustrated, that require an understanding by each player, were one of the main problems identified by participants of the test.

In conceptual terms, the biggest difficulty encountered by participants was the understanding of all the different types of zoning (9 types: Intangible, Primitive...). Therefore, it was highlighted the importance of improving access to information for each player, especially those that explain the different types of zoning. The proposal is to make the players consult with more comfort and efficiency information about the game. For example, it was considered essential that the proposed zoning of each other possible player could always be viewed with the changes visible in "real time" in order to stimulate diversity of strategies for one player, since, that way, each player will be able to see how others players are defending their interests.

The interaction between the players is also a key element in improving SimParc. Considering that the game requires a continuous and dynamic interaction between players, it has been highlighted the importance of the use of flexible systems with additional features such as hyperlinks to send messages directly to a player and use of tools that allow the creation of parallel trading rooms. According to participants, this may facilitate and enhance the negotiation process, an important process in the game.

Aiming to investigate whether SimParc is approaching its epistemic and pedagogical goals, participants were asked about what would be the main goal of the game. The responses were related to the following themes: management practice involving negotiation between different social actors, understanding and experience of different roles that facilitate the learning of the practice of dialogue and negotiation, illustrating the dynamics of conflict, learning environmental expertise and park management, and dissemination of the importance of environmental preservation. In the interpretation of the 
players about if the game had reached such objectives, the players felt that yes, the game was a great exercise for negotiation, with active interaction and interest of players, further encouraged by the possible exchange of roles.

Participants also reported that the main knowledge gained after the game was related to the territorial zoning process of parks, mainly for the players who did not have advanced knowledge on the subject of environmental management. Even those players, who work directly in environmental management, or research related to the subject, explained that they acquired more knowledge about the specific characteristics of each type of zoning commonly used in parks. It was also mentioned that the game could be considered as an exercise on process and techniques of negotiation, although the game does not suggest any technique to the players.

Another point was mentioned in relation to recognition of the diversity of interests in the management of a park. Even though most players knew many of the conflicts illustrated by the game (political, environmental degradation, misuse, etc.), they mentioned that it was possible to improve their analysis based on different roles and groups of social actors that the game presented. Besides the importance of conducting further tests, it was considered an important aspect of the game, the fact that the game is hosted using the Internet, instead of the requirement to install a program on computers, which means greater mobility for applications and larger dissemination of this game.

\section{Park Management Artificial Agent}

As explained in Section 3.1, the park manager acts as an arbitrator in the game, making a final decision for types of conservation for each landscape unit and explains its decision to all players. He may be played by a human or by an artificial agent. The game manager decides when creating and configuring a new game session about the park manager, see Figure 7.

The artificial agent's architecture is structured in two phases. The first decision step concerns agent's individual decision-making process: the agent deliberates about the types of conservation for each landscape unit. Broadly speaking, the park manager agent builds its preference preorder over allowed levels of conservation. An argumentation-based framework (see, e.g. [Dung 1995]) has been implemented to support the decision making. The key idea is to use the argumentation system to select the desires the agent is going to pursue: natural park stakes and dynamics are considered in order to define objectives for which to aim. Hence, decision-making process applies to actions, i.e. levels of conservation, which best satisfy selected objectives. The second step consists in taking account of players' preferences, with the possibility to adjust the profile of the park managers, from autocratic to democratic, and therefore the influence of players' votes. (See details of the complete architecture in [Briot et al. 2009]).

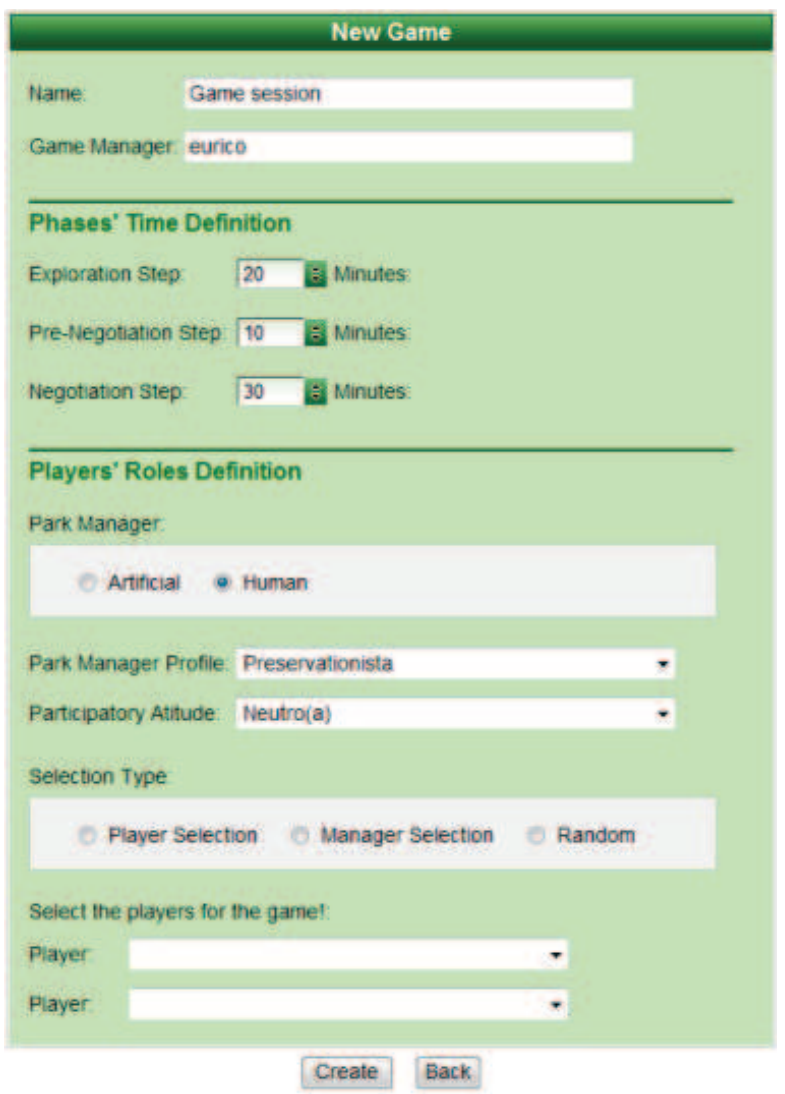

Figure 7: New game configuration interface.

Further details about architecture formal background and implementation are reported in [Briot et al. 2009]. The architecture has been implemented and tested offline and its outputs (decision and arguments) have been validated by our project domain experts. Next step is to organize a new series of game sessions, with an online test of the artificial park manager architecture. Some possible future work is also to use the traces of arguments produced for the decision as a basis for the explanation of the decision to players.

\section{Ongoing Work and Future Prospects}

We are currently planning on inserting other types of artificial agents into the prototype.

\subsection{Artificial Players}

Artificial players represent an ongoing research based on previous experience on virtual players in a computer-supported role-playing game, JogoMan-ViP [Adamatti et al. 2007]. The idea is to possibly replace some of the human players by artificial agents. The two main motivations are: (1) the possible absence of 
sufficient number of human players for a game session and (2) the need for testing in a systematic way specific configurations of players' profiles. The artificial players will be developed along artificial park manager existing architecture (see previous section), with the addition of negotiation and interaction modules. We plan to use the argumentation capabilities to generate and control the negotiation process. In a next stage, we plan to use automated analysis of recorded traces of interaction between human players in order to infer models of artificial players. In some previous work [Guyot and Honiden 2006], genetic programming had been used as a technique to infer interaction models, but we also plan to explore alternative induction and machine learning techniques, e.g., inductive logic programming.

\subsection{Assistant Agents}

The assistant agents are being designed to assist players through the game. It is important to emphasize that the user has total control over his assistant, enabling or disabling it at anytime. The basic initial function of these agents is to present and explain each step of the game. During the negotiation step, assistant agents may also propose to participants some helpful information, in order to improve their analysis concerning the negotiation. For instance, they may provide each player with an ordered list of the players taking into account criteria such as the compatibility or incompatibility of proposals of other players with the proposals of the assisted player. Since we decided to favor a bottom-up approach, we decided to avoid intrusive assistant agents through the game. We believe that intrusive assistant agents could interfere in the players' cognitive processes. That is why our assistant agents cannot suggest players a decision. A first implementation has already been completed and we will soon start to test it through small game sessions.

\subsection{Expert Agents}

Last, we are starting to work on expert agents which will provide the human players (including the park manager if played by a human) with some technical evaluation of the quality and viability of a given park management decision (e.g., considering the survival of an endangered species). Therefore, we plan to identify cases of usage conflicts (e.g., between tourism and conservation of an endemic species) and model the dynamics of the system (in an individual-based/multiagent model or/and in an aggregated model). We would then like to explore the use of viability theory [Aubin 1992] to evaluate possible effects of the decision. These technical evaluations would be encapsulated into expert agents, technical assistants for the players. Another considered type of expert agent will be based on decision theory analysis, for instance to evaluate the dominance relations or equity properties among players votes.

\section{Conclusions}

In this paper, we have presented the SimParc project, a serious game aimed at participatory management of protected areas. We have also summarized the architecture of an artificial decision maker park manager. The first game sessions conducted with domain experts have been successful. It is important to emphasize that the game SimParc was developed based on the recovery of initiatives for the construction of methodologies which help to consolidate democratic spaces of decision in cases of protection of nature. In this sense, the game intends to be a tool capable of contributing to the dialogue on consolidation of commitments to conservation, particularly management of national parks and other protected areas. Although this is an innovative proposal, with wide application in the present context, the experience has shown that quick and simple solutions to modeling the complexity of this process can become a great risk of loss of meaning of the game. Considering that the game could be played too by real managers, it is important to reflect how far the game, that is fun and educational, should be closer to reality and what are the necessary representations/abstractions to achieve the required goals. For example, how the process of negotiating social pacts and democratic management of protected areas can be promoted without losing the focus on respect to real problems and operational by the tax legislation and guidelines for management? Similarly, how to balance technical and scientific expertise in the social participation in the management of nature? Although more evaluation is needed, we believe the initial game session tests are encouraging for the future and we are welcoming any feedback and input from similar or related projects.

\section{Acknowledgements}

The authors would like to thank to all the members of SimParc team and all the participants of game sessions for their help.

This research has been initially funded by the ARCUS Program (French Ministry of Foreign Affairs, Région Ile-de-France and Brazil) and is currently being funded in Brazil by the MCT/CNPq/CT-INFO Grandes Desafios Program (Project No 550865/2007-1) and in France by the Ingénierie Ecologique Program of CNRS \& Cemagref (Project Viabilité SimParc). Some additional individual support is provided by French Ministry of Research (France), AlBan (Europe), CAPES and CNPq (Brazil) PhD fellowship programs.

\section{References}

Adamatti, D., Sichman, J., and Coelho, H. 2007. Virtual players: From manual to semi-autonomous RPG. In Barros, F., Frydman, C., Giambiasi, N., and Ziegler, B., editors, International Modeling and Simulation Multiconference (IMSM'07), Buenos Aires, Argentina, 
The Society for Modeling Simulation International (SCS), February, p. 159-164.

AubIN, J.-P. 1992. Viability theory. Modern Birkhäuser Classics.

BARRETEAU, O. 2003. The joint use of role-playing games and models regarding negotiation processes: characterization of associations. Journal of Artificial Societies and Social Simulation, Vol. 6, No 2.

Briot, JP., Sordoni, A., Vasconcelos, E., Melo, Gustavo AND Irving, M., AND Alvarez, I. 2009. Design of a decision maker agent for a distributed role playing game - Experience of the SimParc project. In Dignum, F., Bradshaw, J., Silverman, B., and van Doesburg, W., editors, Proceedings of the AAMAS Workshop on Agents for Games and Simulations (AGS'09), Budapest, Hungary, AAMAS, May, p. 16-30.

Dung, P. M. 1995. On the acceptability of arguments and its fundamental role in non-monotonic reasoning, logic programming and n-person games. Artificial Intelligence, 77(2):321-357.

Guyot, P. and Honiden, S. 2006. Agent-based participatory simulations: Merging multiagent systems and roleplaying games. Journal of Artificial Societies and Social Simulation, 9(4).

IRVING, M. (Org.). 2006. Áreas Protegidas e Inclusão Social: Construindo Novos Significados. Rio de Janeiro: Aquarius.

KolB, D.A. 1984. Experimental learning: Experience as the source of learning and development. Prentice Hall.

Michael, D. and Chen, S. 2006. Serious Games - Games that Educate, Train and Inform. Thomson Course Technology.

Vasconcelos, E., Briot JP., Irving, M., Barbosa, S., FuRTADO, V. 2009. A user interface to support dialogue and negotiation in participatory simulations. In Nuno David and Jaime Simão Sichman, eds, Multi-AgentBased Simulation IX - International Workshop, MABS 2008, Estoril, Portugal, May 12-13, 2008, Revised and Invited Papers, LNAI, No 5269, Springer-Verlag, p. 127140.

Warmerdam J., Knepflé M., Bidarra R., Bekebrede G. AND MAYER I. 2006. Sim-Port: a multiplayer management game framework. In Proceedings of the 9th International Conference on Computer Games (CGAMES'06), Dublin, Ireland. 